\title{
Nano-Science and Nano-metrology for Societal Benefits
}

\author{
T. J. B. M. Janssen and D. Roy* \\ National Physical Laboratory, Hampton Road, Teddington TW11 0LW, UK \\ Published online: 28 December 2013 \\ (C) Metrology Society of India 2013
}

In general nanotechnology can be described as the manipulation of matter with at least one dimension in the range of 1-100 $\mathrm{nm}$. It refers to a very broad range of research and applications whose common trait is size. It includes fields of science as diverse as surface science, organic chemistry, molecular biology, semiconductor physics, quantum physics, etc. The applications of nanotechnology are equally broad, ranging from devices for ICT to completely new concepts of molecular self-assembly, from intelligent coatings to super-strong materials with designed properties, and of course microfabrication.

The concept of nanotechnology was probably first discussed by Richard Feynman in his famous talk "There is plenty of room at the bottom", in which he described the possibility of synthesis (assembling) via direct manipulation of atoms. There have also been a number of Nobel Prizes in this field. The first one, in 1986, was for the invention of the scanning tunnelling microscope in 1981 by Binning and Rohrer at IBM. This invention kick-started the whole field of scanning probe microscopy which enabled the study of physics at the atomic scale. In 1996 Kroto and Smalley received the Nobel Prize for their discovery of the molecule $\mathrm{C}_{60}$-fullerene (a perfect sphere of 60 carbon atoms) in 1985. As recently as 2010, Geim and Novoselov from the University of Manchester received the Nobel Prize for their 2004 discovery of graphene, a single atomic layer isolated from graphite. In particular graphene has sparked an unparalleled burst of scientific activity across the globe with the number of scientific publications exceeding 40,000.

The present and future societal impact of nanotechnology is also huge. Nanotechnology can be found in thousands of products already publicly available, from sunscreens and cosmetics to food packaging and surface coatings. Also the electronics industry is awash with nano-

*Corresponding author, E-mail: debdulal.roy@npl.co.uk components. The transistor gate length in the latest computer chips is as small as $10 \mathrm{~nm}$ and in these devices quantum effects such as tunnelling start to dominate the performance. Maybe more interesting are the future applications of nanotechnology such as the development of personalised healthcare, and energy generation and storage which have the potential to dramatically change our society. Quite possibly the biggest applications are the ones we haven't even thought of yet.

Realising this potential brings us to the need for metrology to underpin this technology. Metrology has a crucial role in order to produce nanomaterials and devices with a high degree of accuracy and reliability in nanomanufacturing. The central challenge in nanometrology is to develop new measurement techniques, reference samples and standards which meet the future requirements of industry. Already, the need for measurement and characterisation of some new devices exceeds the current capabilities of metrology laboratories and will become even more pressing in the near future. The metrology community needs to respond to this and continue to drive down the minimum length scale in measurement science. This activity will require fundamental research which often will involve collaborations with academia as well as close collaboration with end-users of nanotechnology that are responsible for product development and manufacturing. Because the functioning of nanotechnology devices critically relies on dimensions and surface dependant properties, metrology will form an essential component in the fabrication process and the challenge is to achieve such high-end measurements away from the metrology laboratory on the factory floor.

In this special issue of MAPAN we have aimed to cover several aspects from this wide spectrum of nano-science and nano-metrology that includes quantum metrology, surface and nanoanalysis, nanofabrication, solar cell and standardisation of nanotechnologies. We hope this special issue will bring an insight into the needs for metrology and 
standards for safe and sustainable development of nanotechnology and realise its enormous benefit to our society.



T. J. B. M. Janssen was born in the Netherlands in 1965 . He received the MS and $\mathrm{PhD}$ degrees in physics from the University of Nijmegen, The Netherlands, in 1989 and 1994, respectively. His doctoral work was on the far-infrared magneto-optical properties of low dimensional semiconductor structures and organic conductors. From 1994 to 1998, he was a Research Fellow at the University of Bristol, Bristol, UK, investigating the Fermi surface properties of heavy Fermion metals and superconductors using the de Haas-van Alphen effect. JT has been at NPL since 1998, he is responsible for the research on quantum electrical standards. JT is an NPL Fellow and a Fellow of the Institute of Physics.



D. Roy received his B.Tech in Engineering from the Indian Institute of Technology, Kharagpur in 1997 and $\mathrm{PhD}$ from the University of Cambridge, UK in 2002. He spent the next two years at the Nanoscience Centre, Cambridge developing tip-enhanced Raman spectroscopy as a nanoscale chemical characterisation tool. His current research interest at the National Physical Laboratory, UK includes Surface and Nanoanalysis of soft materials development of the related standards. 